\title{
The Impact of Vascular Loops in the Cerebellopontine Angle on Audio-Vestibular Symptoms: A Systematic Review
}

\author{
Anna-Maria Papadopoulou ${ }^{a} \quad$ Nikolaos Bakogiannis $^{b} \quad$ Valentinos Sofokleous $^{a}$ \\ Ioanna Skraparic Chris Bakoyiannis ${ }^{\mathrm{b}}$ \\ aDepartment of Otolaryngology, Athens General Children's Hospital "Pan. \& Aglaia Kyriakou", Athens, Greece; \\ ${ }^{b}$ First Department of Surgery, Laikon General Hospital, Athens, Greece; 'First Department of Internal Medicine, \\ Evangelismos General Hospital, Athens, Greece
}

\section{Keywords}

Cerebellopontine angle · Anterior inferior cerebellar artery · Microvascular compression · Vestibulocochlear nerve . Hearing loss · Tinnitus

\begin{abstract}
It has been suggested that vascular loops in the cerebellopontine angle and internal auditory canal are involved in the etiology of audio-vestibular symptoms. Several studies have focused on the compression of the eighth cranial nerve by vascular loops but have yielded contradictory results regarding their clinical significance. The aim of this study was to investigate whether vascular loops in this region correlate with audio-vestibular symptoms and which loop features - if any - can potentially lead to symptom manifestation. This systematic review was conducted according to the PRISMA guidelines. We performed on PubMed a literature search from November 2005 to October 2020. The search strategy included the following keywords ("vascular loops" OR "AICA loops" OR "vascular compression syndrome") AND ("hearing loss" OR "tinnitus" OR "vertigo"). Fifteen studies were eligible and included in the analysis. Overall, the studies encompassed a total of 11,788 patients included in this review. The
\end{abstract}

significantly larger group of patients (70\%), in which no correlation of symptoms with vascular loops was found, suggests that vascular loops are probably anatomic variations in a substantial majority of cases with an uncommon subset causing some audio-vestibular symptoms. Even within the papers claiming a correlation, there is a multitude of symptoms that did not correlate with vascular loops. It has been suggested by most authors that magnetic resonance imaging should be performed to exclude the role of a vascular loop in the etiology of audio-vestibular symptoms only when vascular compression syndrome is suspected based on clinical indications and not routinely. Further studies would be useful in order to detail the relationship between the vascular structures and the nervous system.

(c) 2022 S. Karger AG, Basel

\section{Introduction}

Although various diseases are associated with otologic symptoms such as tinnitus, the cause is not identified in almost $60 \%$ of patients [Ensari et al., 2017]. In some cases, it is believed that the etiology involves a vascular loop in the anterior inferior cerebellar artery (AICA), insinuating 
itself into the internal auditory canal (IAC) [De Abreu et al., 2016]. The term vascular compression syndrome, which refers to a group of diseases caused by direct contact between a blood vessel and a cranial nerve, was introduced by McKenzie in 1936 and popularized by Jannetta in 1975 [Jannetta, 1975]. It has been suggested as a potential cause of hearing deficit, typewriter tinnitus, and equilibrium disturbance or vertigo [Bae et al., 2017], and it may be screened by abnormal results in neuro-otologic tests [Beyazal Celiker et al., 2017]. Imaging of the cerebellopontine angle (CPA) is highly valuable in diagnosing the etiology of this group of diseases. High-resolution thin-section magnetic resonance imaging (MRI) is a widely used technique for visualizing the IAC and the CPA in patients with auditory symptoms and provides critical clues about the anatomical relationships between the vestibulocochlear nerve (CVN) and surrounding vasculature in the temporal bone [Beyazal Celiker et al., 2017]. The prevalence of vascular loops that affect the VIII nerve in the root entry zone, where the nerve exits from the CPA and is not covered by myelin sheet, and the IAC is extremely variable and ranges from 7 to $23 \%$ [Di Stadio et al., 2020]. On the other hand, vascular loops can be identified in many asymptomatic individuals [Gorrie et al., 2010]. Although numerous articles have focused on this condition, the existence of vascular compression syndrome continues to be questioned [De Abreu et al., 2016]. Several authors have examined the relationship between vascular loops in the posterior cranial fossa and the IAC and neuro-otologic symptoms; however, whether symptoms can be predicted by specific features of vascular loops is mostly still unclear [Di Stadio et al., 2020]. Given the existing discrepancy in the literature, the aim of this study was to investigate whether vascular loops in this region correlate with audio-vestibular symptoms and which loop features - if any - can potentially lead to symptom manifestation.

\section{Anatomy Background}

The CPA is bordered superiorly by the medial portion of the midbrain. This is the region where some important structures, including the trigeminal nerve, facial nerve, and CVN, exit the brain [Gorrie et al., 2010]. After the merging of the vestibular and cochlear root, the trunk of the CVN leaves the brain through the posterior cranial fossa, traveling lateral to the abducens nerve and the facial nerve. The CVN then extends anteriorly and laterally. Together with the facial and the intermediate nerve, it enters

The Impact of Vascular Loops on Audio-

Vestibular Symptoms the IAC. At the fundus of the IAC, the nerve splits into its two roots, vestibular and cochlear [Gultekin et al., 2008]. Cerebellar floccules hide the root entry zone of the CVN [Sirikci et al., 2005]. The other important anatomical structure that is discussed in this article is the AICA. This artery along with the cerebellopontine fissure vein is located in the middle or cerebellopontine fissure of the $\mathrm{CPA}$. The AICA traverses near the abducens, facial nerve, and CVN [Sirikci et al., 2005]. It can originate from the basilar artery $(98.1 \%)$ or from the vertebral artery $(1.9 \%)$ as a single $(92.3 \%)$ or duplicate $(7.7 \%)$ artery [Sirikci et al., 2005]. It passes the IAC outside the meatus $(19 \%-$ $40 \%)$, at the meatus (33\%-56\%), or within the IAC (25\%27\%) [Van der Steenstraten et al., 2007]. Vascular loops are anatomical abnormalities that arise from the branches of the basilar artery (98\%) or from the vertebral artery (2\%) [Ensari et al., 2017]. Most authors grade the vascular loops according to the Chavda classification, which is based on the anatomical position of the AICA loop in relation to the IAC and was recommended by McDermott et al. [2003]: grade I - when an AICA vascular loop borders the IAC; grade II - when the loop enters IAC but does not extend more than $50 \%$ of the length of the IAC; and grade III - when the loop occupies more than $50 \%$ of the canal [McDermott et al., 2003]. Another classification system was proposed by Sirikci et al. [2005] and was used in other studies as well. It includes four different types of anatomical relationship observed between the AICA and CVN. In type 1 (point compression), the AICA compresses only a limited portion of the CVN. In type 2 (longitudinal compression), the AICA approaches the CVN as both traverse parallel to each other. In type 3 (loop compression), the vascular loop of the AICA encircles the CVN. In type 4 (indentation), the AICA compresses the $\mathrm{CVN}$ so as to make an indentation in the nerve [Sirikci et al., 2005].

\section{Symptoms}

The clinical significance of these anatomical variants, according to some authors, include symptoms such as sensorineural hearing loss (SNHL), tinnitus, and vertigo. Particular types that belong to the spectrum of these symptoms are mentioned below. First, sudden SNHL (SSNHL) is defined as $30 \mathrm{~dB}$ SNHL at three consecutive audiometric frequencies with a rapid onset occurring in a 72-h period. Although many factors, such as infectious, vascular, immunological, metabolic, neoplastic, ototoxic, and traumatic are believed to cause damage to the cochlea, in most cases, the exact cause still remains unknown [Ezerarslan et al., 2017]. Additionally, vestibular 


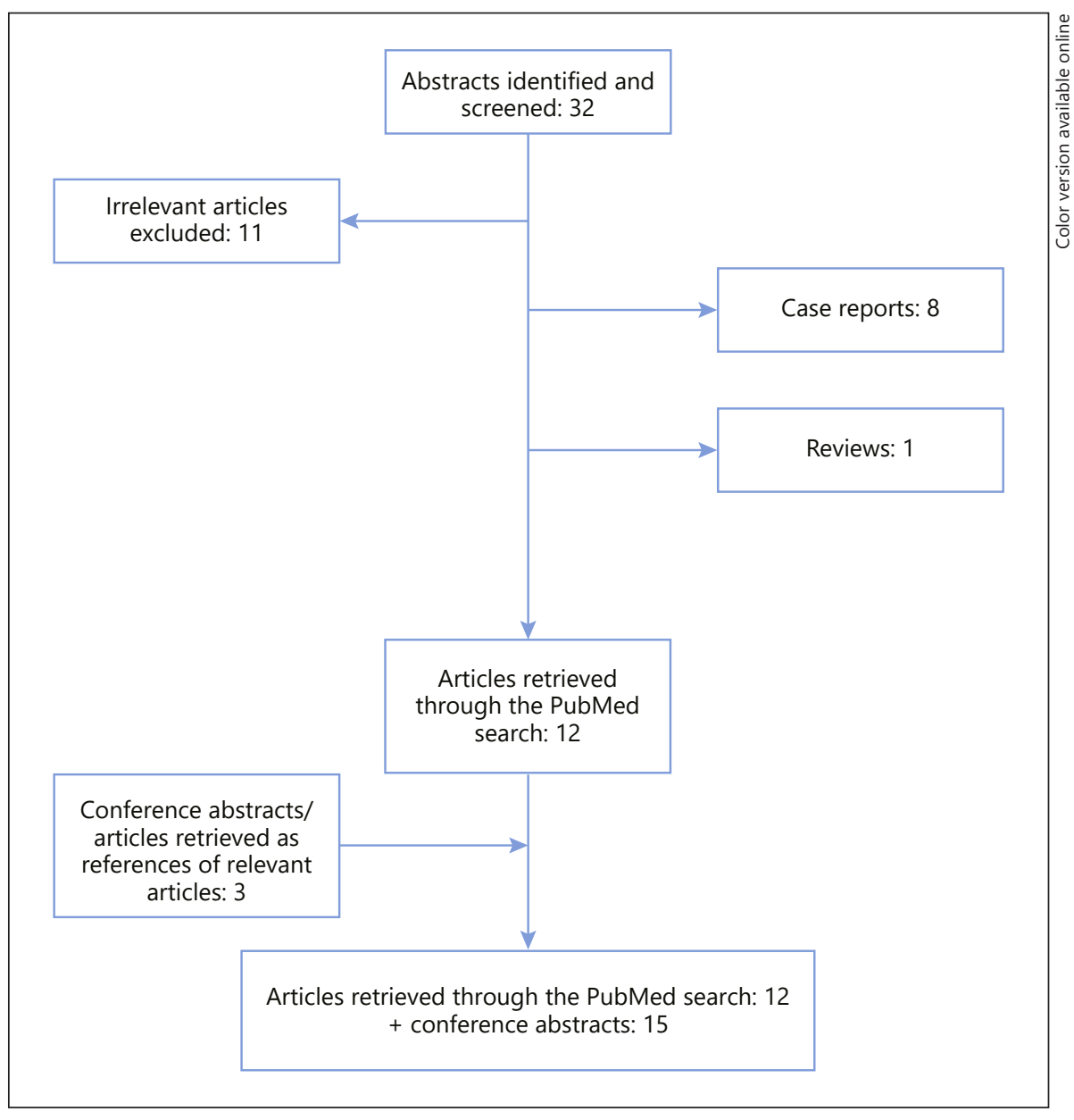

Fig. 1. Flowchart of the search strategy.

paroxysmia is also discussed in one study. In this condition, vertigo entity typically presents with short, sharp spells of vertigo and periodic (but not compulsory) nausea and has a recurrent nature. In up to $60 \%$ of paroxysmia cases, vertigo spells might be induced by certain head movement. Over time, patients seem to develop a caloric vestibular function loss [Loader et al., 2016].

\section{Methods}

This systematic review was performed in accordance with the PRISMA guidelines. Eligible articles were identified by a search of the PubMed bibliographical database for the period from November 2005 up to October 2020. The study protocol was agreed by all coauthors. The search strategy included the following keywords ("vascular loops" OR "AICA loops" OR "vascular compression syndrome”) AND ("hearing loss" OR "tinnitus" OR "vertigo"). Language restrictions were applied (only articles in English, French, and German were considered eligible); two investigators (A.M.P. and N.B.), working independently, searched the literature and extracted data from each eligible study. Reviews were not eli- gible, while all prospective and retrospective studies, as well as case reports, were eligible for this systematic review. Manuscripts that did not state the names of the authors were excluded. In addition, we checked all the references of relevant reviews and eligible articles that our search retrieved, so as to identify potentially eligible conference abstracts. Titles of interest were further reviewed by abstract. Finally, reference lists of eligible studies were manually assessed in order to detect any potential relevant article ("snowball" procedure).

\section{Results}

\section{Article Selection and Study Demographics}

The search strategy retrieved 42 articles that were evaluated for full-text evaluation. Studies based on radiologic investigations were regarded as the most applicable to clinical practice. Fifteen studies were deemed eligible and were included in the analytic cohort. Overall, studies encompass a total of 11,788 patients that have been included in this systematic review. The search strategy is depicted in Figure 1. 
Table 1. Correlation between vascular loops and cochleovestibular symptoms numbers in bold represent statistically significant correlations ( $p$ value $<0.05$ )

\begin{tabular}{|c|c|c|c|}
\hline First author, year & $N$ & Symptoms & $p$ value \\
\hline \multirow[t]{3}{*}{ Sirikci et al., 2005} & \multirow[t]{3}{*}{140} & Vertigo & $>0.05$ \\
\hline & & Dizziness & $>0.05$ \\
\hline & & Tinnitus & $>0.05$ \\
\hline Van der Steenstraten et al., 2007 & 167 & Hearing loss & $>0.05$ \\
\hline Gultekin et al., 2008 & 102 & Tinnitus & $>0.05$ \\
\hline \multirow[t]{2}{*}{ Gorrie et al., 2010} & \multirow[t]{2}{*}{458} & Hearing loss & 0.016 \\
\hline & & Tinnitus & 0.043 \\
\hline Cho et al., 2012 & 156 & Tinnitus & $<0.05$ \\
\hline \multirow[t]{5}{*}{ Hoekstra et al., 2015} & \multirow[t]{5}{*}{321} & Tinnitus & $>0.05$ \\
\hline & & Hearing loss & 0.22 \\
\hline & & Otalgia & 0.18 \\
\hline & & Vertigo & 0.48 \\
\hline & & Hemifacial spasm & 0.85 \\
\hline \multirow[t]{3}{*}{ De Abreu et al., 2016} & \multirow[t]{3}{*}{33} & Tinnitus & 0.838 \\
\hline & & Hearing loss & 0.693 \\
\hline & & Dizziness & 0.568 \\
\hline \multirow[t]{3}{*}{ Loader et al., 2016} & \multirow[t]{3}{*}{119} & Tinnitus & $>0.05$ \\
\hline & & Hearing loss & $>0.05$ \\
\hline & & Vestibular neuritis & $<0.001$ \\
\hline Ezerarslanet al., 2016 & 106 & Sudden hearing loss & 0.017 \\
\hline Ensari et al., 2017 & 44 & Tinnitus & 0.481 \\
\hline Bae et al., 2017 & 23 & Tinnitus & 0.032 \\
\hline Beyazal Celiker et al., 2017 & 417 & Vertigo & $>0.05$ \\
\hline \multirow[t]{3}{*}{ Di Stadio et al., 2019} & \multirow[t]{3}{*}{2,622} & Vertigo & 0.002 \\
\hline & & Tinnitus & 0.003 \\
\hline & & Hemifacial spasm & $<0.001$ \\
\hline Li et al., 2019 & 6,978 & Hearing loss + tinnitus & 0.321 \\
\hline \multirow[t]{4}{*}{ Mejia-Quiñones et al., 2020} & \multirow[t]{4}{*}{102} & Tinnitus & $<0.05$ \\
\hline & & Hearing loss & $>0.05$ \\
\hline & & Nystagmus & $>0.05$ \\
\hline & & Vertigo & $>0.05$ \\
\hline
\end{tabular}

\section{Main Findings}

Table 1 illustrates the correlation between the presence of vascular loops and audio-vestibular symptoms. Many studies have been performed with little success in demonstrating a significant link between eighth nerve contact by vascular loops and symptom manifestation. In particular, Li et al. [2019] performed a statistical correlation between the laterality of the vascular loop in the IAC/CPA as graded according to the Chavda classification and the laterality of audio-vestibular symptoms in 6,978 patients, but the level of significance was not reached [Li et al., 2019]. Another retrospective study as- sessed the clinical relevance of potentially found AICA loops and found no significant relationships between the presence of an AICA loop and the side of the tinnitus, abnormalities on the ABR, or complaints specific to nerve compression syndrome [Hoekstra et al., 2015]. Although Ensari et al. [2017] found various types of vascular loop, no statistically significant relationship was determined between the presence of vascular loop and tinnitus as stated by the Tinnitus Handicap Inventory scores [Ensari et al., 2017]. Gultekin et al. [2008] also investigated the causative effect of the vascular loop and compression of the CVN at the CPA in patients with unex- 
plained tinnitus but found no statistically significant differences between the patient and control groups for the anatomic type of vascular loop, the vascular contact, and the angulation of the CVN at the CPA [Gultekin et al., 2008]. In addition, Van der Steenstraten et al. [2007] measured the length and width of the IAC and found no association between a narrow meatus, the presence of an AICA loop, and the development of a microvascular compression syndrome in $167 \mathrm{MRI}$ scans of patients with unexplained unilateral SNHL [Van der Steenstraten et al., 2007]. Neither did Sirikci et al. [2005]manage to find statistically significant relationship between audio-vestibular symptoms including tinnitus, vertigo, and hearing loss and any type of compression of the CVN by AICA loop in 140 patients [Sirikci et al., 2005]. According to Beyazal Celiker et al. [2017] MRI findings were similar for the patients with and without vertigo, resulting in no clinical relationship of vertigo symptoms with the presence of vascular loops in the CPA and IAC [Beyazal Celiker et al., 2017]. Finally, De Abreu et al. [2016] compared ears that presented vascular loops with those that did not and found no association with tinnitus, hearing loss, or vertigo. Similarly, they found no association between the Chavda grade and any otological symptom [De Abreu et al., 2016].

Although the rest of the collected studies have reported opposite results, it appears that AICA loops are not associated with all audio-vestibular symptoms. For instance, according to Cho et al. [2012], ears with type I and II AICA loops and ears with a thinner AICA loop showed significantly higher rates of tinnitus. These authors found no association between the type of tinnitus and hearing loss [Cho et al., 2012]. A retrospective, multicenter study by Di Stadio et al. [2020] analyzed 2,622 consecutive MRI scans of the CPA of patients with asymmetric audio-vestibular symptoms. Vascular loops in direct nerve contact correlated with vertigo, tinnitus, and hemifacial spasm, while asymmetric SNHL correlated with the number of contacts and length of contact. These authors also found that the cochlear nerve was more commonly involved in vascular impingements than vestibular and facial nerves, which could explain why the majority of the collected studies include patients who present more commonly with hearing symptoms than vertigo or hemifacial spasm [Di Stadio et al., 2020]. Furthermore, Ezerarslan et al. [2017] evaluated patients with idiopathic sudden SNHL and concluded that the loop type of branching regarding the AICA was identified significantly more commonly in patients than in healthy controls. Moreover, these patients are unresponsive to standard oral corticosteroid therapy more than the other branching types [Ezerarslan et al., 2017]. Likewise, Gorrie et al. [2010] studied structural features of vascular loops formed by the AICA within the CPA and IAC and tried to correlate them with unexplained asymmetric hearing loss. They found no statistically significant association between loops classified by the Chavda system and hearing loss. Statistically significant association was also not present between loops that made no contact with the nerve, ran adjacent to the nerve, or displaced the nerve. Yet, a statistically significant association was found between loops that ran between the facial nerve and CVN and hearing loss. The subset that had tinnitus in addition to hearing loss had similar results [Gorrie et al., 2010]. According to another study, an association was observed between vascular loops and tinnitus, while no associations were found as far as SNHL, nystagmus, or vertigo were concerned [Mejía-Quiñones et al., 2020]. Additionally, Loader et al. [2016] compared the different types of neurovascular contact between the CVN and the AICA in patients presenting with clinical signs of acute vestibular neuritis with and without subsequent objective vestibular function loss. They showed a significantly higher number of neurovascular contact and vascular loops in patients presenting with symptoms of acute peripheral vestibulopathy without objective vestibular function loss compared to patients with pathological caloric irrigation results and healthy controls, suggesting a possible connection between the presence of vascular loops and acute vestibular dysfunction [Loader et al., 2016]. Finally, Bae et al. [2007] examined whether radiologic evidence of neurovascular compression of the cochlear nerve is pathognomonic in typewriter tinnitus. The comparison of the anatomic location of AICA loops in the IAC and CPA among symptomatic sides and healthy controls did not yield statistically significant differences but showed a trend toward the incidence of a type III AICA loop being higher in symptomatic sides. Nevertheless, the type of contact of the CVN by the AICA loop was significantly different between the above groups. However, due to considerable false-positive and false-negative radiologic findings, they suggest that other clues such as meticulous history taking and response to carbamazepine should be more reliable than radiologic evidence of neurovascular contact [Bae et al., 2017].

Examining the number of studied patients in the analyzed papers, the number of patients represented in each of the opposing groups of papers is significantly different. In particular, $69.57 \%(8,202 / 11,788)$ of patients showed no correlation of vascular loops with audio-vestibular 
symptoms. This does not consider those noncorrelated symptoms in the papers with selected audio-vestibular symptoms found to be correlated with vascular loops.

\section{Discussion}

Although the concept of vascular contacts at the root exit zone of the facial, trigeminal, and glossopharyngeal nerves have been widely accepted as a cause of hemifacial spasm, trigeminal, and glossopharyngeal neuralgia, respectively, its relationship with otologic symptoms such as tinnitus, hearing loss, and dizziness is not clear yet [De Abreu et al., 2016; Ensari et al., 2017; Ezerarslan et al., 2017]. The concept of vascular compression syndromes was popularized by Jannetta, who reported a reduction in dysfunctional hyperactivity of the eighth cranial nerve after using microsurgery to separate the nerve from a blood vessel in patients with intractable vertigo, supporting the theory that an arterial loop is a factor of dysfunction [Jannetta, 1975; Jannetta, 1997]. The most common anatomical structures that cause compression of the VCN are AICA, posterior inferior cerebellar artery, and/or their branches [Beyazal Celiker et al., 2017]. On the basis of that study, various other researchers have attempted to establish a relationship between vascular compressions and clinical conditions, such as hearing loss, vertigo, and tinnitus. Other studies have advocated this concept by performing microvascular decompression in patients suspected of having audio-vestibular neurovascular compression syndrome and showing favorable clinical outcomes with good hearing thresholds after the operation [Bae et al., 2017]. On the other hand, medical treatment aims to reduce tinnitus through the use of drugs such as carbamazepine [Levine, 2006].

However, controversy remains regarding the pathophysiology of these conditions. Different theories have been suggested, including focal demyelination due to contact between vessel and nerve, disturbances in the distribution of blood flow resulting in reduced vascular perfusion, or direct transmission of pulsations to the cochlea via the petrous bone [Nowe et al., 2004; De Abreu et al., 2016; Di Stadio et al., 2020). As far as vestibular symptoms are concerned, both neuronal excitation and progressive neuronal damage due to direct contact between the blood vessel and nerve have been proposed as possible mechanisms [Hufner et al., 2008; Loader et al., 2016]. Still, most studies fail to prove any significant correlation with vascular loops. This may be due to the high redundancy of the vestibular system which controls balance by

The Impact of Vascular Loops on AudioVestibular Symptoms relying on a number of different mechanisms involving the visual and motor system [Thompson and Amedee, 2009].

It is of note that during the last decades, MRI has been the preferred imaging method for the diagnosis of vascular compression syndrome in patients with auditory-vestibular symptoms due to its high sensitivity and specificity rates [Kazawa et al., 2013]. Volumetric sequences with strong T2 weighting present advantages over conventional angiographic examinations, given that, in addition to being noninvasive (not involving the use of contrast), the former allow the assessment of the blood vessels, as well as of the nerve in question and the harmonic or potentially pathological relationship between the two [Satoh et al., 2007].

A persistent unanswered question regards the relationship between radiologic evidence of neurovascular compression and audio-vestibular symptoms. The major concern is that AICA loops are frequently found in the IAC even in asymptomatic healthy subjects, which has been demonstrated by cadaveric studies as well [Gorrie et al., 2010; De Abreu et al., 2016]. This is why many authors consider vascular loops as an anatomic variation rather than a causal radiological finding. MRI, as mentioned above, provides excellent delineation of the neurovascular structures in the IAC and CPA. This could explain the higher rates of vascular loops than those found previously in the literature, as well as the fact that many radiologic studies have reported a comparable incidence of neurovascular contact between the AICA and the CVN in symptomatic and asymptomatic subjects [Bae et al., 2017; Beyazal Celiker et al., 2017]. Moreover, it has been proposed that neurovascular contact syndrome is caused by demyelination of the cochlear nerve due to the proximity of the AICA loop and not necessarily by direct contact. As a result, radiologically demonstrable neurovascular contact without any symptoms may occur. Conversely, based on the theory that neurovascular contact is dynamic in some patients, meaning that the contact is on and off, MRI may have only captured the "contact-off" status [Bae et al., 2017].

In fact, microvascular compression is often treated as a diagnosis of exclusion since it lacks a clear diagnostic test, and patients with this syndrome are often diagnosed with another condition [Gorrie et al., 2010]. Notably, certain radiologic findings can increase the likelihood that an otoneurological symptom is related to neurovascular compression. Such findings include distortion of the nerve (either longitudinal bending or cross-sectional deformation or both) caused by a vessel, vascular com- 
pression at the emergence of the nerve root, and a perpendicular intersection between a blood vessel and a nerve [Naraghi et al., 2007; Van der Steenstraten et al., 2007].

The wide discrepancies among the studies may be explained by different methodology; for example, a control group was not used in all studies. Moreover, some studies used all consecutive symptomatic patients, while others used all consecutive MRI scans and tried to correlate these to clinical findings. In addition, observer differences can alter the results of estimation or evaluation of the various types of audio-vestibular diseases in different studies [Sirikci et al., 2005].

\section{Conclusion}

The significantly larger group of patients $(70 \%)$ in which no correlation of symptoms with vascular loops was found suggests that vascular loops are probably anatomic variations in a substantial majority of cases with an uncommon subset causing some audio-vestibular symptoms. Even within the papers claiming a correlation, there is a multitude of symptoms that did not correlate with vascular loops. In fact, it has been suggested by most authors that an MRI should be performed to exclude a vascular loop, based on specific clinical indications and not routinely. Further multicentric randomized controlled studies would be useful in order to detail the relationship between the vascular structures and the nervous system, perhaps generating new classifications that increase the power to discriminate between truly pathological cases and those within the limits of normality and also focusing on the intracranial part of the eight cranial nerve [Van der Steenstraten et al., 2007; De Abreu et al., 2016]. Moreover, since abnormal auditory brain stem response (ABR) findings have been suggested to be diagnostic of cochlear nerve compression, future studies including $A B R$ for the initial evaluation of patients should be performed to further evaluate the value of ABR as a diagnostic tool [De Ridder et al., 2007].

\section{Statement of Ethics}

An ethics statement is not applicable because this study is based exclusively on published literature.

\section{Conflict of Interest Statement}

The authors have no conflicts of interest to declare.

\section{Funding Sources}

The authors received no financial support for the research and authorship of this article.

\section{Author Contributions}

All the authors have provided substantial contributions to the conception or design of the work or the interpretation of data for the work (A.P., N.B., V.S., I.S., and C.B.). All of them worked on the draft or revised it critically for important intellectual content. The final version was approved for publishing by all the authors. The authors agree on accountability for all aspects of the work in ensuring that questions related to the accuracy or integrity of any part of the work are appropriately investigated and resolved.

\section{Data Availability Statement}

All data generated or analyzed during this study are included in this article. Further inquiries can be directed to the corresponding author.

\section{References}

Bae YJ, Jeon YJ, Choi BS, Koo JW, Song JJ. The role of MRI in diagnosing neurovascular compression of the cochlear nerve resulting in typewriter tinnitus. Am J Neuroradiol. 2017 Jun;38(6):1212-7.

Beyazal Celiker F, Dursun E, Celiker M, Durakoglugil T, Beyazal M, Inecikli MF, et al. Evaluation of vascular variations at cerebellopontine angle by 3D T2WI magnetic-resonance imaging in patients with vertigo. J Vestib Res. 2017;27(2-3):147-53
Cho YH, Lee SH, Park C, Min HJ, Choi EJ, Oh J, et al. The association of anterior inferior cerebellar artery in IAC with tinnitus and hearing loss. Otolaryngol Head Neck Surg. 2012; 147(2):222-3.

De Abreu L Jr, Kuniyoshi CH, Wolosker AB, Borri ML, Antunes A, Ota VK, et al. Vascular loops in the anterior inferior cerebellar artery, as identified by magnetic resonance imaging, and their relationship with otologic symptoms. Radiol Bras. 2016 Sep-Oct;49(5): 300-4.
De Ridder D, Heijneman K, Haarman B, van der Loo E. Tinnitus in vascular conflict of the eighth cranial nerve: a surgical pathophysiological approach to ABR changes. Prog Brain Res. 2007;166:401-11.

Di Stadio A, Dipietro L, Ralli M, Faralli M, Della Volpe A, Ricci G, et al. Loop characteristics and audio-vestibular symptoms or hemifacial spasm: is there a correlation? A multiplanar MRI study. Eur Radiol. 2020 Jan;30(1):99109. 
Ensari N, Gür ÖE, Selçuk ÖT, Renda L, Osma Ü, Eyigör $\mathrm{H}$, et al. Is presence of vascular loop in magnetic resonance imaging always related to tinnitus? J Craniofac Surg. 2017 May;28(3): 295-8.

Ezerarslan H, Sanhal EO, Kurukahvecioğlu S, Ataç GK, Kocatürk S. Presence of vascular loops entering internal acoustic channel may increase risk of Sudden sensorineural hearing loss and reduce recovery of these patients. Laryngoscope. 2017 Jan;127(1):210-5.

Gorrie A, Warren FM 3rd, de la Garza AN, Shelton $\mathrm{C}$, Wiggins RH 3rd. Is there a correlation between vascular loops in the cerebellopontine angle and unexplained unilateral hearing loss? Otol Neurotol. 2010 Jan;31(1):48-52.

Gultekin S, Celik H, Akpek S, Oner Y, Gumus T, Tokgoz N. Vascular loops at the cerebellopontine angle: is there a correlation with tinnitus? AJNR Am J Neuroradiol. 2008 Oct; 29(9):1746-9.

Hoekstra CE, Prijs VF, van Zanten GA. Diagnostic yield of a routine magnetic resonance imaging in tinnitus and clinical relevance of the anterior inferior cerebellar artery loops. Otol Neurotol. 2015 Feb;36(2):359-65.

Hüfner K, Barresi D, Glaser M, Linn J, Adrion C, Mansmann U, et al. Vestibular paroxysmia: diagnostic features and medical treatment. Neurology. 2008 Sep;71(13):1006-14.

Jannetta PJ. Neurovascular cross-compression in patients with hyperactive dysfunction symptoms of the eighth cranial nerve. Surg Forum. 1975;26:467-9.
Jannetta PJ. Outcome after microvascular decompression for typical trigeminal neuralgia, hemifacial spasm, tinnitus, disabling positional vertigo, and glossopharyngeal neuralgia (honored guest lecture). Clin Neurosurg. 1997;44:331-83.

Kazawa N, Togashi K, Ito J. The anatomical classification of AICA/PICA branching and configurations in the cerebellopontine angle area on 3D-drive thin slice T2WI MRI. Clin Imaging. 2013 Sep-Oct;37(5):865-70.

Levine RA. Typewriter tinnitus: a carbamazepine-responsive syndrome related to auditory nerve vascular compression. J Otorhinolaryngol Relat Spec. 2006;68(1):43-7.

Li L, Amiraraghi N, Begbie F, Kontorinis G. The significance of vascular loops in the internal auditory meatus: a true incidental imaging finding? Eur Arch Otorhinolaryngol. 2019 Dec;276(12):3275-80.

Loader B, Linauer I, Korkesch S, Krammer-Effenberger I, Zielinski V, Schibany N, et al. A connection between neurovascular conflicts within the cerebellopontine angle and vestibular neuritis, a case controlled cohort study. Acta Otorhinolaryngol Ital. 2016 Oct;36(5): 421-7.

McDermott AL, Dutt SN, Irving RM, Pahor AL, Chavda SV. Anterior inferior cerebellar artery syndrome: fact or fiction. Clin Otolaryngol Allied Sci. 2003 Apr;28(2):75-80.

Mejía-Quiñones V, Valderrama-Chaparro JA, Paredes-Padilla S, Orejuela-Zapata JF, Granados-Sánchez AM. Vascular loop in the cerebellopontine angle: clinical-radiological correlation. Radiologia. 2020 Oct 8:S00338338(20)30113-2.
Naraghi R, Tanrikulu L, Troescher-Weber R, Bischoff B, Hecht M, Buchfelder M, et al. Classification of neurovascular compression in typical hemifacial spasm: three-dimensional visualization of the facial and the vestibulocochlear nerves. J Neurosurg. 2007 Dec; 107(6):1154-63.

Nowé V, De Ridder D, Van de Heyning PH, Wang XL, Gielen J, Van Goethem J, et al. Does the location of a vascular loop in the cerebellopontine angle explain pulsatile and nonpulsatile tinnitus? Eur Radiol. 2004 Dec; 14(12):2282-9.

Satoh T, Onoda K, Date I. Fusion imaging of three-dimensional magnetic resonance cisternograms and angiograms for the assessment of microvascular decompression in patients with hemifacial spasms. J Neurosurg. 2007 Jan;106(1):82-9.

Sirikci A, Bayazit Y, Ozer E, Ozkur A, Adaletli I, Cüce MA, et al. Magnetic resonance imaging based classification of anatomic relationship between the cochleovestibular nerve and anterior inferior cerebellar artery in patients with non-specific neuro-otologic symptoms. Surg Radiol Anat. 2005 Dec;27(6):531-5.

Thompson TL, Amedee R. Vertigo: a review of common peripheral and central vestibular disorders. Ochsner J. 2009;9(1):20-6.

Van der Steenstraten F, de Ru JA, Witkamp TD. Is microvascular compression of the vestibulocochlear nerve a cause of unilateral hearing loss? Ann Otol Rhinol Laryngol. 2007 Apr; 116(4):248-52. 